\title{
Rhinoscleroma causing upper airway obstruction
}

\author{
Geetika Verma MD ${ }^{1}$, D Kanawaty MD FRCPC ${ }^{2}$, R Hyland MD FRCPC $^{3}$
}

\begin{abstract}
G Verma, D Kanawaty, R Hyland. Rhinoscleroma causing upper airway obstruction. Can Respir J 2005;12(1):43-45.
\end{abstract}

\section{Rhinosclérome en cause dans une obstruction respiratoire}

\begin{abstract}
Le rhinosclérome est une maladie granulomateuse chronique du tractus respiratoire qui n'est pas rare sous les tropiques, en particulier au Mexique, en Amérique centrale et au Moyen-Orient. Quelques cas ont été signalés en Amérique du Nord, affectant principalement des immigrants provenant des pays touchés. L'agent causal est Klebsiella rhinoscleromatis, un coccobacille gram-négatif. Le diagnostic se fonde sur la mise en culture de l'agent pathogène et la présence caractéristique des cellules de Mikulicz au microscope optique.

La maladie affecte principalement les voies respiratoires supérieures et s'accompagne d'écoulement nasal, d'obstruction nasale et de douleur frontale. Malgré le terme « rhinosclérome », l'appareil respiratoire entier peut être atteint. Bien qu'elle progresse lentement, la maladie évolue souvent vers une destruction importante. L'atteinte laryngotrachéale s'observe chez environ 15 à $80 \%$ des cas, mais elle est rarement isolée. Dans l'article actuel, on décrit un cas de rhinosclérome accompagné de symptômes d'obstruction des voies respiratoires supérieures.
\end{abstract}

Rhinoscleroma is a chronic granulomatous condition of the respiraMexico, Central America and the Middle East. A few cases have been reported in North America, primarily involving immigran rhinosic countries. The causative organism is Klebsiella the basis of culture of the organism and the characteristic pathology of Mikulicz cells on light microscopy.

The condition primarily affects the upper airway, and frequently pres ents with nasal discharge, nasal obstruction or frontal facial pain. Despite the term 'rhinoscleroma', there may be involvement of the entire respiratory tract. Although the condition is slowly progressive, its natural course portends extensive destruction. Laryngotracheal involvement occurs in approximately $15 \%$ to $80 \%$ of cases, but patients rarely present with isolated laryngotracheal disease. In the present paper, a case of rhinoscleroma presenting with symptoms of upper airway obstruction is described.

Key Words: Laryngotracheal scleroma; Rhinoscleroma; Upper airway obstruction

\begin{abstract}
A 56-year-old male pharmacist, originally from Egypt, preAsented with a two-month history of shortness of breath on exertion. He complained of a foreign-body sensation in his throat with associated wheezing but denied chest pain, cough, hemoptysis or hoarseness. He had not experienced fever, weight loss or night sweats.

His past medical history was significant for chronic sinusitis since childhood, characterized by long-standing malodourous secretions, crusting and intermittent nasal blockage. He had seen an otolaryngologist and received a tentative diagnosis of immotile cilia syndrome 12 years before his current presentation, although this diagnosis was not proven by biopsy and he was the natural father of two children. He had both hypertension and hyperlipidemia, and no known family history of respiratory illness or malignancy. He quit smoking six weeks before presentation and had a history of one pack/week for 20 years.

On physical examination, he appeared well with no evidence of respiratory distress at rest. His blood pressure was $140 / 90 \mathrm{mmHg}$. His trachea was midline and he did not have cervical or supraclavicular adenopathy, cyanosis or clubbing.
\end{abstract}

Examination of his chest revealed symmetric chest expansion with normal breath sounds throughout all lung fields, and specifically, an absence of stridor or wheezing. The remainder of his physical examination was normal.

A chest $\mathrm{x}$-ray taken at the time of presentation was normal, with no evidence of a mass or parenchymal disease. He had had an x-ray six years previously which appeared the same.

He underwent spirometry and produced a flow-volume loop demonstrating classical features of fixed upper airway obstruction (Figure 1).

A computed tomography scan of the patient's thorax revealed normal lungs with wall thickening of the proximal trachea and an irregular-appearing endoluminal surface at the level of the thyroid. A subsequent computed tomography scan of the head and neck showed moderate circumferential narrowing of the subglottic larynx and superior trachea, as well as nodular thickening at the level of the inferior cricoid resulting in $50 \%$ to $60 \%$ endoluminal narrowing (Figure 2 ).

The patient underwent bronchoscopy, the findings of which were suggestive of a proximal tracheal tumour involving

\footnotetext{
${ }^{1}$ University of Toronto, Department of Medicine; ${ }^{2}$ North York General Hospital, Division of Respirology, Department of Medicine;

${ }^{3}$ St Michael's Hospital, Division of Respirology, Department of Medicine, Toronto, Ontario

Correspondence and reprints: Dr R Hyland, St Michael's Hospital, 30 Bond Street, Toronto, Ontario M5B 1W8. Telephone 416-864-5810,

fax 416-864-6034, e-mail hylandb@smh.toronto.on.ca
} 


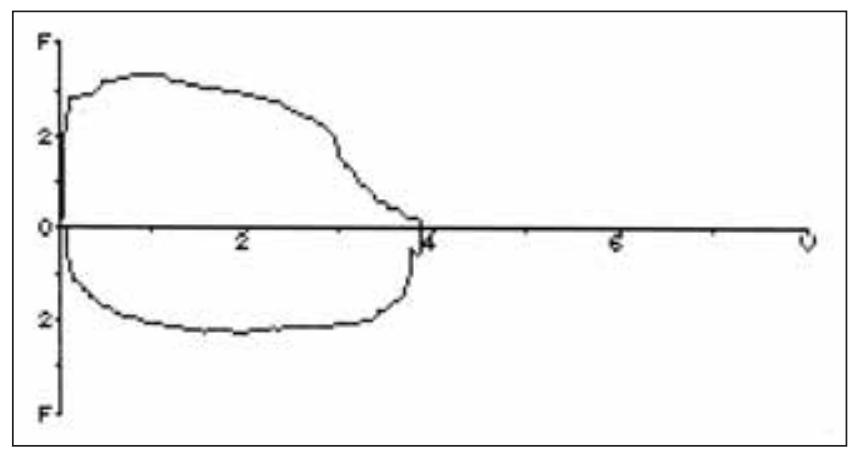

Figure 1) Maximum inspiratory and expiratory flow-volume curve demonstrating flattening of the inspiratory and expiratory flow loops, which is consistent with fixed upper airway obstruction
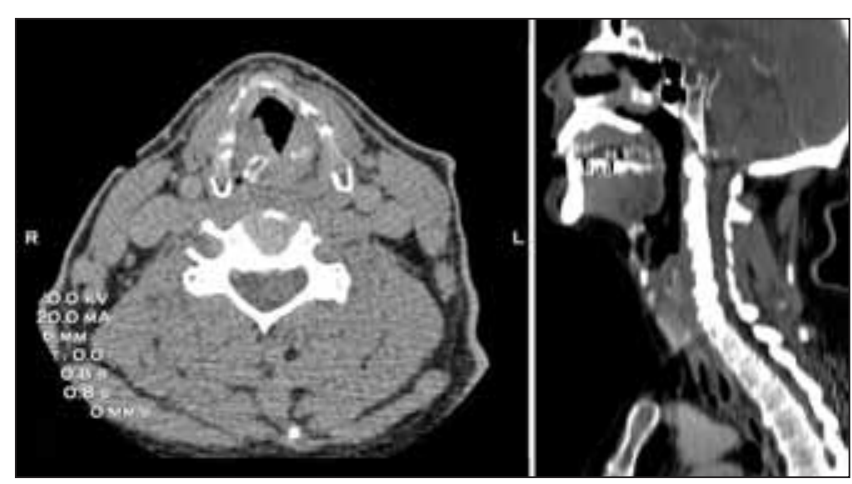

Figure 2) Selected axial (left) and sagittal (right) views from the computed tomography scan of the head and neck demonstrating a subglottic endoluminal mass extending to the superior trachea

the glottis and subglottis. The patient was referred to an otolaryngologist for evaluation of a potential malignancy. Laryngoscopy confirmed that there was a $3 \mathrm{~cm}$ tumour of the tracheal lumen, with evidence of cartilaginous destruction. The mid- and distal trachea were clear, as were the supraglottic and glottic larynx. Endoscopic resection was performed to improve the patient's airway and biopsies were sent for pathology.

Pathology revealed a packed mucosal infiltrate of histiocytes admixed with inflammatory cells (mainly plasma cells). Silver methenamine and Gram staining revealed numerous Gram-negative bacilli within the histiocytes. The low-power view of the laryngeal biopsy showed an infiltrate of large, vacuolated histiocytes or Mikulicz cells (Figure 3).

Culture of the tumour specimen confirmed Klebsiella rhinoscleromatis was the causative organism. The patient was started on ciprofloxacin hydrochloride $500 \mathrm{mg}$ twice a day for six weeks. He remained well after resection with no symptoms of upper airway obstruction. Bronchoscopic follow-up at six months demonstrated no recurrence of disease.

\section{DISCUSSION}

The objective of the present case discussion is to highlight a disease process that is generally identified predominantly as a cause of nasal disease, although it can involve the lower respiratory tract. Because it is not endemic to North America and infrequently presents to respiratory specialists, the diagnosis may be missed. However, with increasing immigration from

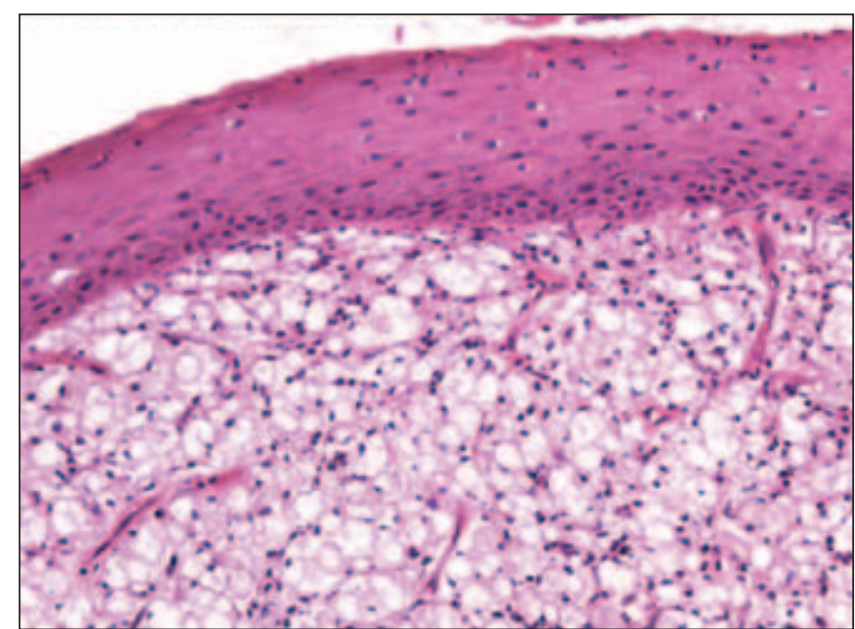

Figure 3) Low-power view of the laryngeal biopsy demonstrating the characteristic vacuolated histiocytes or Mikulicz cells beneath the squamous epithelial layer (hematoxylin and eosin stain; original magnification $\times 200$ )

endemic countries, this cause of upper airway disease should be familiar to Canadian respirologists. In the present case, the patient described a long-standing history of nasal obstructive symptoms and rhinorrhea before his presentation to a respirologist. He remained misdiagnosed and untreated until his disease progressed to cause proximal tracheal obstruction. Ultimately, the diagnosis was not made on the basis of patient presentation or appearance of the lesion, but rather on the basis of the pathological findings and culture. If suspicion of malignancy is sufficiently high and the characteristic pathology is missed, Gram staining may not be performed and the causative organism may not be identified.

Typically, rhinoscleroma progresses through three clinical stages. Initially, a catarrhal phase is characterized by purulent and fetid rhinorrhea, which may progress to nasal mucosal atrophy and crusting. The second phase is a granulomatous phase, in which nodules or masses may lead to obstructive symptoms in the respiratory tract. The final stage is a cicatricial stage of scarring which may cause external deformity or stenosis of nasal passages or laryngeal airway (1).

The differential diagnoses for respiratory scleroma include nasal tumours and other tumours of the respiratory tract, fungal infections or leprosy. Wegener's granulomatosis, T cell lymphomas, sarcoidosis and basal cell carcinomas may also have similar clinical presentations.

Alfaro-Monge et al (2) described a case of scleroma presenting as an upper tracheal mass causing stridor and respiratory distress. The patient was managed with urgent resection and an eight-week course of oral ciprofloxacin hydrochloride. In their literature review of lower respiratory tract scleroma, there were only two cases of isolated laryngotracheal scleroma, both of which required urgent tracheotomy $(3,4)$. In a Los Angeles study of 22 patients with scleroma (5), 59\% of patients had laryngotracheal scleroma. All patients in this study had nasal mucosal involvement. Patients presented with dysphonia, exertional dyspnea and/or stridor. Patients in this series were initially managed with intravenous antibiotics and dexamethasone, and three required tracheotomies. All patients were in the granulomatous phase of infection, and it appeared that granuloma formation was more common in the glottis than in 
the subglottis. Yigla et al (6) described a case of a severe form of laryngotracheal rhinoscleroma, which resulted in the death of the patient.

The mainstay of treatment is surgical debridement, when indicated for preservation of the airway, and prolonged antibiotic therapy (7-9). Agents of choice include quinolones, doxycycline or tetracycline, and less frequently, streptomycin and rifampicin $(8,9)$. Based on reported cases $(8)$, several weeks of therapy are indicated because the relapse rate is high. The present patient had a six-week course of ciprofloxacin hydrochloride and has exhibited a satisfactory clinical response after six months of follow-up. Long-term follow-up is indicated to ensure remission on the basis of both clinical and endoscopic findings.

ACKNOWLEDGEMENTS: The authors would like to thank Dr Pratibha Iyengar from the University of Toronto, Department of Pathology.

\section{REFERENCES}

1. Fajardo-Dolci G, Chavolla R, Lamadrid-Bautista E, Rizo-Alvarez J. Laryngeal scleroma. J Otolaryngol 1999;28:229-31.

2. Alfaro-Monge JM, Fernandez-Espinosa J, Soda-Merhy A. Scleroma of the lower respiratory tract: Case report and review of literature. J Laryngol Otol 1994;108:161-3.

3. Agarwal MK, Samant HC, Gupta OP, Gupta S. Solitary scleroma of the larynx. Ear Nose Throat J 1981;60:315-7.

4. Jay J, Green RP, Lucente FE. Isolated laryngeal rhinoscleroma. Otolaryngol Head Neck Surg 1985;93:669-73.

5. Amoils CP, Shindo ML. Laryngotracheal manifestations of rhinoscleroma. Ann Otol Rhinol Laryngol 1996;105:336-40.

6. Yigla M, Ben-Izhak O, Oren I, Hashman N, Lejbkowicz F. Laryngotracheobronchial involvement in a patient with nonendemic rhinoscleroma. Chest 2000;117:1795-8.

7. Badia L, Lund VJ. A Case of rhinoscleroma treated with ciprofloxacin. J Laryngol Otol 2001;115:220-2.

8. Bhargava D, Date A. Palatal presentations of scleroma. J Laryngol Otol 2001;115:679-80.

9. Ammar ME, Rosen A. Rhinoscleroma mimicking nasal polyposis. Ann Otol Rhinol Laryngol 2001;110:290-2. 


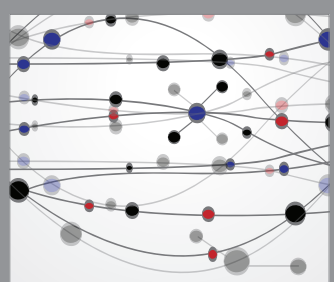

The Scientific World Journal
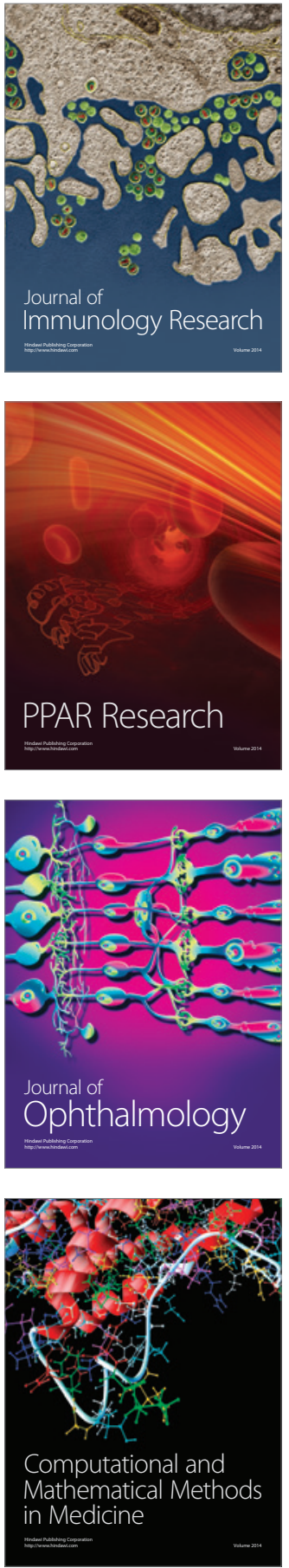

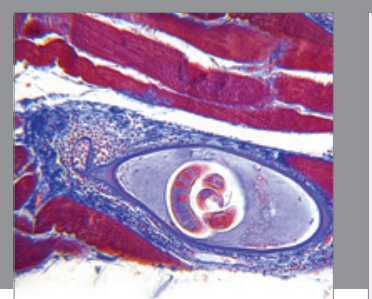

Gastroenterology Research and Practice

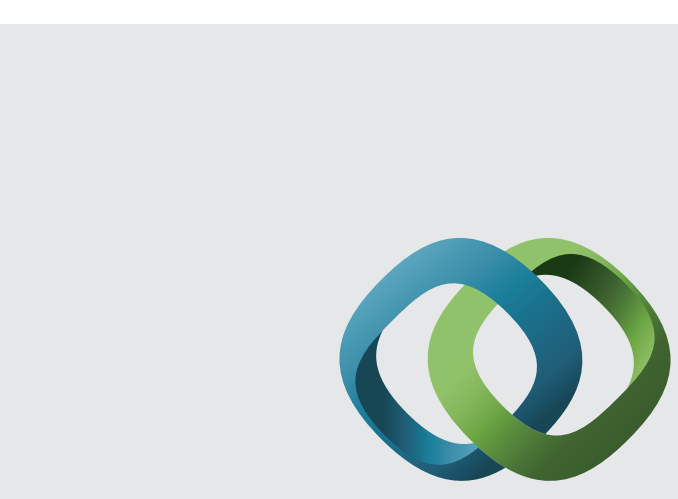

\section{Hindawi}

Submit your manuscripts at

http://www.hindawi.com
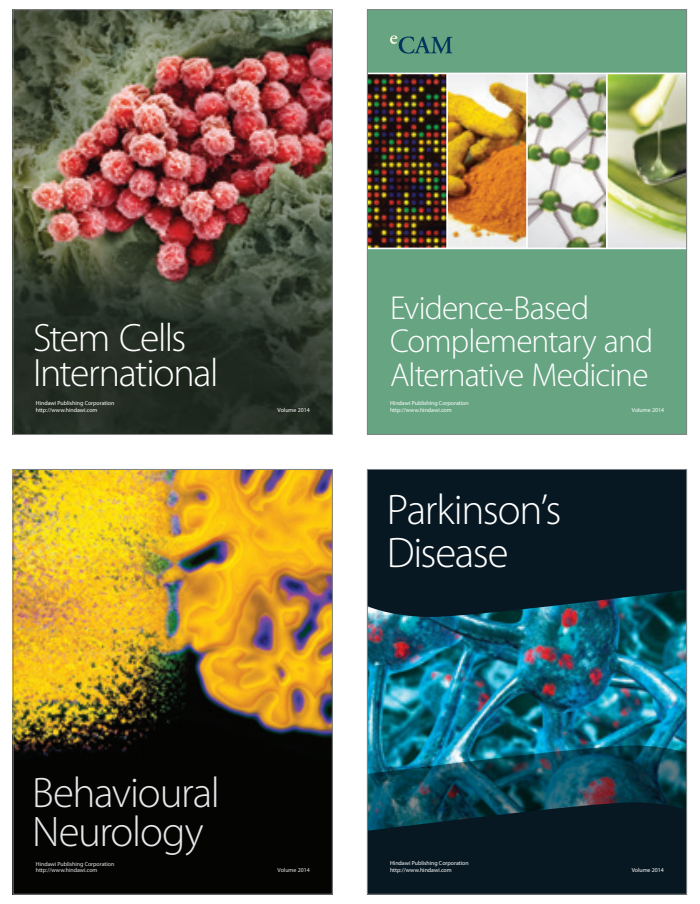
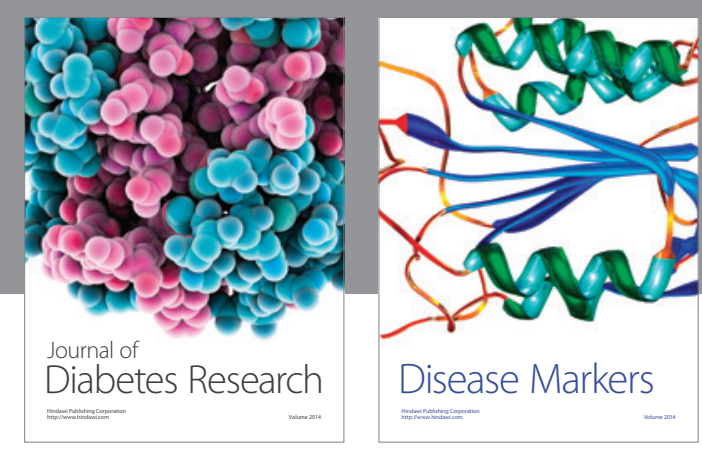

Disease Markers
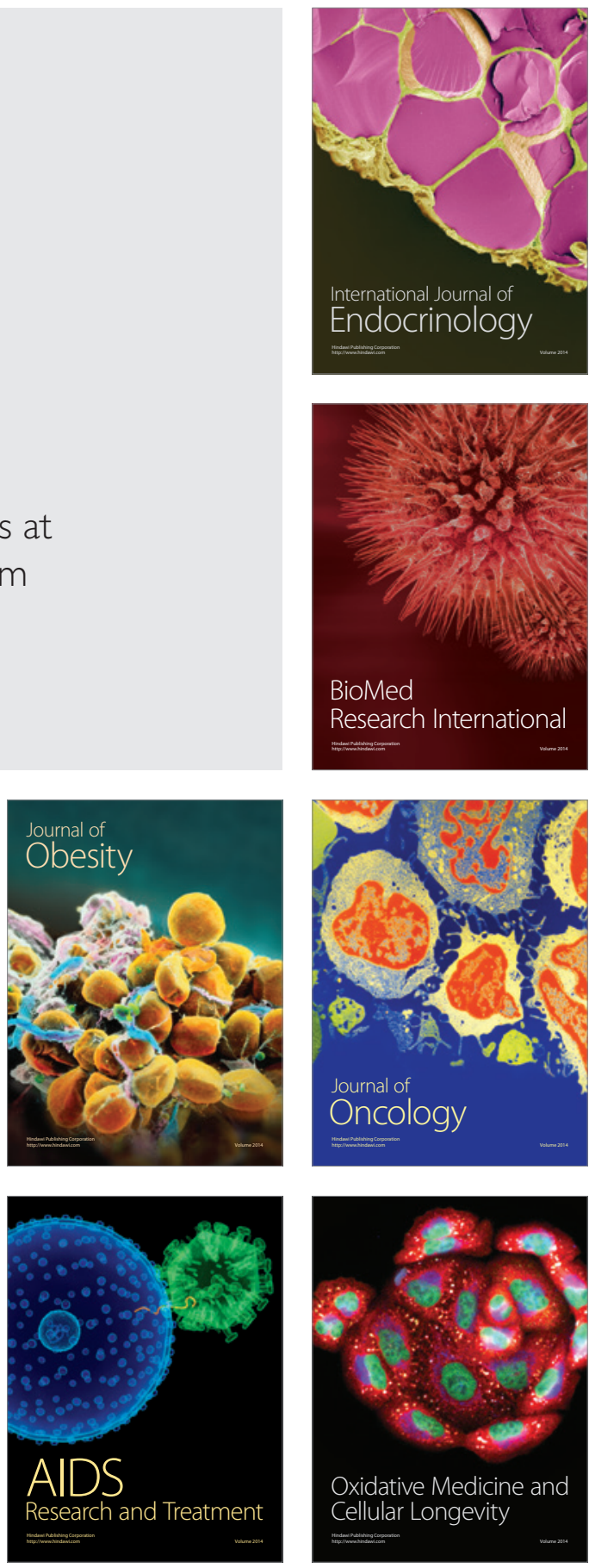